\title{
Invasion pinning in a periodically fragmented habitat
}

James Dowdall (Department of Mathematics and Statistics, University of Ottawa)

Victor LeBlanc (Department of Mathematics and Statistics, University of Ottawa)

Frithjof Lutscher (Department of Mathematics and Statistics, and Department of Biology, University of Ottawa)

\begin{abstract}
Biological invasions can cause great damage to existing ecosystems around the world. Most landscapes in which such invasions occur are heterogeneous. To evaluate possible management options, we need to understand the interplay between local growth conditions and individual movement behaviour. In this paper, we present a geometric approach to studying pinning or blocking of a bistable travelling wave, using ideas from the theory of symmetric dynamical systems. These ideas are exploited to make quantitative predictions about how spatial heterogeneities in dispersal and/or reproduction rates contribute to halting biological invasion fronts in reaction-diffusion models with an Allee effect. Our theoretical predictions are confirmed using numerical simulations, and their ecological implications are discussed.
\end{abstract}

\section{Introduction}

Biological invasions are the cause of great concern worldwide. Alien species that establish and spread in new habitats may alter an existing ecosystem and, for example, cause loss of biodiversity. Hence, it is important that we understand the mechanisms that prevent or facilitate the invasion of an alien species. While many mechanisms of growth, dispersal and spread in homogeneous environments are well studied and understood (Fife and McLeod, 1977; Hadeler and Rothe, 1975; Volpert, Volpert, and Volpert, 1994; Weinberger, 1982), the question of how spatial heterogeneity affects the invasion process is a currently active research area with many questions still open. Most existing landscapes are heterogeneous and many intervention measures against biological invasions are necessarily local in space, increasing existing heterogeneity (With, 2002). Since spatial heterogeneity may influence not only a species' growth rate but also its dispersal ability and behaviour, we need to understand how these processes interact to allow an invasive species to spread. When the species' growth function does not exhibit an Allee effect, i.e. when the per capita growth rate decreases with density, many important insights can and have been gained from 
studying linearization of the nonlinear model at low population density (Shigesada, Kawasaki, and Teramoto, 1986; Maciel and Lutscher, 2013; Musgrave and Lutscher, 2014; Weinberger, 2002; Berestycki, Hamel, and Nadirashvili, 2005; Freidlin and Gartner, 1979). When there is an Allee effect, traditional analytical techniques for determining the invasion speed fail, but numerical simulation and homogenization have provided first insights (Maciel and Lutscher, 2015; Musgrave, Lutscher and Girard, 2015; Xin, 2002; Ding, Hamel and Zhao, 2017; Heinze, 2001). In this work, we extend a novel technique, based on equivariant bifurcation theory (LeBlanc and Roy, 2013), to study wave propagation failure in such a way that it can be applied to mathematical models for spatial spread with Allee effect in a heterogeneous landscape.

Reaction-diffusion equations (RDEs) are among the primary mathematical tools used in studying biological invasions (Volpert, Volpert, and Volpert, 1994; Shigesada and Kawasaki, 1997). Traditionally, RDEs take the form

$$
\frac{\partial u}{\partial t}=D \frac{\partial^{2} u}{\partial x^{2}}+\mathcal{F}(u),
$$

where $u(x, t)$ denotes the density of organisms at location $x$ and time $t, D$ is the diffusion coefficient and $\mathcal{F}(u)$ is the reaction term that describes the population growth dynamics.

RDEs allow us to incorporate individual-level growth and movement behaviour to make analytical predictions about the rate of spatial spread of a population. For example, the logistic growth function $\mathcal{F}(u)=\rho u(1-u)$ in the FisherKPP model results in the "asymptotic spreading speed" $c^{*}=2 \sqrt{\mathcal{F}^{\prime}(0) D}=$ $2 \sqrt{\rho D}$ (Weinberger, 1982). A locally introduced population will spread at this minimal rate. In addition, for each $c \geq c^{*}$ there exists a monotone traveling wave solution $u(x, t)=U(x+c t)$ of equation (1).

Many species exhibit an Allee effect, i.e. an increase of the per-capita growth rate with density as long as total density is low enough (Allee, 1949; Courchamp, Berec, and Gascoigne, 2008). A strong Allee effect, where population growth is actually negative below a certain threshold, can be modelled by the function

$$
\mathcal{F}(u)=\rho u(1-u)(u-\alpha),
$$

where $\alpha$ is the Allee threshold (Keitt, Lewis and Holt, 2001), and $\rho$ is a scaling parameter related to the time scale and the maximum growth rate. In this particular case, the asymptotic spreading speed is given by Hadeler and Rothe (1975)

$$
c^{*}=\sqrt{2 \rho D}\left(\frac{1}{2}-\alpha\right) .
$$

In contrast to the Fisher-KPP case above, a traveling wave solution exists only for this particular speed. The profile of this traveling wave is given explicitly by $u(x, t)=u^{*}(x+c t)$ where

$$
u^{*}(x)=\frac{1}{2}\left(1+\tanh \left(\frac{x}{2 \sqrt{2}}\right)\right) .
$$


From the explicit expression for $c^{*}$ in (3), we see that the invasion advances when $\alpha<1 / 2$, retreats when $\alpha>1 / 2$ and stops when $\alpha=1 / 2$. For purposes of managing an invasive pest species, it is therefore this threshold value at $\alpha=1 / 2$ which is important, since it delineates these two cases. At the precise value $\alpha=1 / 2$, we say that there is "invasion pinning". Of course, in nature, we can not expect that $\alpha$ will have precisely this value of $1 / 2$, so absent any inhomogeneity, we will observe either an advancing or a retreating invasion front. Keitt, Lewis and Holt (2001) have shown showed that the situation changes in a spatially discrete landscape. In a linear array of identical patches with nearestneighbour diffusion, there is a range of values of $\alpha$ (depending on $D$ ) for which an invading species is 'pinned', i.e. it neither spreads nor retreats.

In this work, we study invasion pinning in a continuous but spatially heterogeneous habitat. Keener and Lewis (2000) have shown that a single, localized spatial heterogeneity can stop the propagation of a spreading action potential in a neural model. By applying comparison principles, they argued that conditions that guarantee the existence of a steady state correspond to a necessary condition to stop propagation.

We consider a spatially periodic habitat that represents the fragmented landscape that one increasingly finds in nature where natural processes and human activities create patches of habitat of different quality (With, 2002). In an idealized scenario, the diffusion and reaction terms in equation (1) are periodic functions of spatial location (with the same period). The study of spreading phenomena in such periodically fragmented landscapes began with the work by Shigesada, Kawasaki, and Teramoto (1986) and has since been generalized substantially, but typically assumes that there is no Allee effect. First steps to study RDEs in periodic habitats with Allee effect are given by Maciel and Lutscher (2015); Ding, Hamel and Zhao (2015); Xin (1993); Nadin (2015), and for integrodifference equations by Musgrave, Lutscher and Girard (2015).

Maciel and Lutscher (2015) used homogenization techniques to find an approximation of the spreading speed in a periodic landscape of period $L$ in the limit of small $L$, i.e. when the inhomogeneity rapidly oscillates. For example, when $\tilde{\rho}(x)$ is a periodic function of period 1 , then the function $\tilde{\rho}(x / L)$ is $L$ periodic. If $\tilde{\rho}$ has arithmetic average $\langle\tilde{\rho}\rangle$, then one can expect that, in the limit as $L \rightarrow 0$, the spreading speed of the heterogeneous equation with $\rho=\tilde{\rho}(x / L)$ is approximately that of the homogeneous equation with $\rho=\langle\tilde{\rho}\rangle$. In particular, invasion pinning occurs only for a single parameter value (or a very narrow range). However, they noticed that when $L$ is not small, the approximation can be very poor and invasion pinning can occur for a relatively large range of parameter values. Musgrave and Lutscher (2014) studied invasion pinning in a discrete-time model and gave some estimates for when pinning occurs when $L$ is not small. Similarly, Keener (2000) observed that the behaviour of the homogenized system and the exact system are not the same.

To illustrate this effect, we numerically solved equation (1) with Allee function (2) and periodic growth function

$$
\rho(x)=1+\varepsilon \sin (x) .
$$


The Allee threshold parameter is fixed at $\alpha=0.4646<0.5$ while $\varepsilon$ is varied. According to (3), the invasion succeeds in the homogeneous landscape when $\varepsilon=0$. The two simulations in Figure 1 show that the wave still propagates when $\varepsilon$ is small but invasion pinning occurs when $\varepsilon$ is larger.
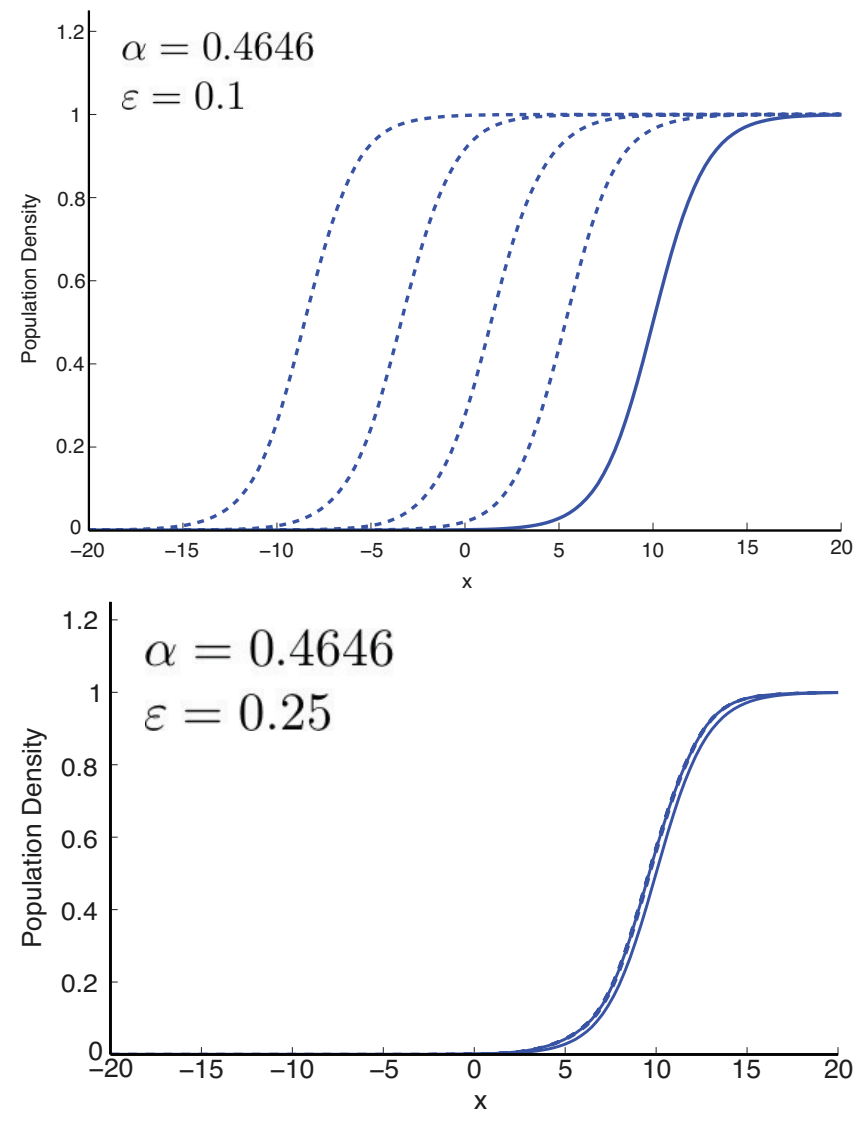

Figure 1: Successive snapshots of a solution of equations (1), (2) and periodic function (5). The initial wave profile is given by a solid line while the successive snapshots taken at regular time intervals, are given by the dashed lines. Parameters are $\alpha=0.4646$, and $\varepsilon=0.1$ (top), $\varepsilon=0.25$ (bottom). All numerical integrations of RDEs in the paper are done using an explicit (in time) finitedifference scheme with Neumann boundary conditions at the edges. The spatial step size is $\Delta x=0.03$ and the time step size is $\Delta t=0.1$. The profiles are taken at every 100 time units $(1000 \Delta t)$.

Keener (2000) proposed an alternative approach to homogenization by carefully examining the averaging theorem. Our alternative approach is based on and extends the theory of symmetry-breaking perturbations developed by LeBlanc and Roy (2013) and by Roy (2013). We present the necessary back- 
ground of this theory in the next section. Their formulation considers a perturbation in the reaction term. We extend their results in such a way that the perturbation can be included in the diffusion term as well. This extension is highly relevant in ecological applications since individuals do adjust their movement behaviour to spatially changing landscape attributes; please see Maciel and Lutscher (2013) and reference therein. Any attempt to quantify conditions that would pin an invasion need to account for movement behaviour. We give the proof of our main theorem in appendix A. We illustrate the theory with several examples in section 3 before we close with a discussion.

\section{Theory and Results}

In this section, we will provide the main mathematical results required for our analysis of the effects of spatial heterogeneities on propagation failure. Note that by "propagation failure" in this paper, we mean that the wave is stalled.

The results rest mostly on group-equivariant bifurcation theory, and are adapted from LeBlanc and Roy (2013) and Roy (2013) for our purposes. Since the details are rather technical, we divide this section into 2 subsections: the first gives a qualitative heuristic description of the main ideas of our methods, and the second gives the proper theorem statements and technical details.

\subsection{Forced symmetry-breaking for travelling waves}

RDEs such as (1) possess a very simple but important symmetry property: whenever $u(x, t)$ is a solution of this RDE, then so is $v(x, t):=u(x+a, t)$, for any constant $a \in \mathbb{R}$. This is a consequence of the translation invariance of the second derivative operator $\frac{\partial^{2}}{\partial x^{2}}$, and the fact that the nonlinear reaction term $\mathcal{F}(u)$ does not depend explicitly on $x$.

If we view (1) as a dynamical system on an appropriate infinite-dimensional space of functions $Y$, then the group $\mathrm{SE}(1)$ of all rigid translations (which is essentially the real numbers with addition) acts on $Y$ as follows: for all $a \in \operatorname{SE}(1)(\cong \mathbb{R})$ and for all $U \in Y, a$ maps $U$ to an element $\mathcal{T}_{a} U \in Y$, where $\left(\mathcal{T}_{a} U\right)(x)=U(x+a)$.

Consequently, solutions to (1) are organized in group orbits: applying any translation to a solution of (1) yields another solution of (1). In general, there is no reason to expect that these two group-conjugate solutions are on the same time orbit of the dynamical system (1), although it is possible that they are. Namely, travelling waves are exactly such kinds of solutions: the SE(1) group orbit of a travelling wave solution of (1) coincides with (or contains) its time orbit for the dynamical system generated by (1). In the terminology of equivariant dynamical systems, we call such solutions relative equilibria.

As we can see in (2), (3) and (4), for the bistable RDE, the relative equilibria are parametrized by the Allee parameter $\alpha$ (or equivalently by $c$ via formula (3)). 


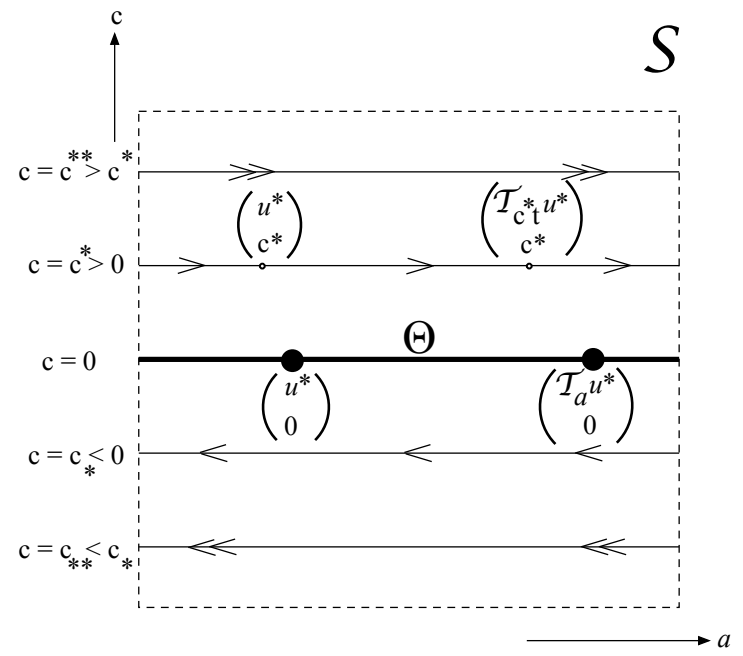

Figure 2: Dynamics of all travelling wave solutions to (1) in the bistable case $(2)$ on the two-dimensional invariant center manifold $\mathcal{S}$. This flow is described by the planar system of ODEs $\dot{a}=c, \dot{c}=0$. On each horizontal line, the flow evolves linearly in time at speed $c$. The line $\Theta$ at $c=0$ is therefore a line of equilibria, which correspond to the phenomenon known as propagation failure.

Figure 2 gives a geometric depiction and summarizes how these travelling wave solutions are organized as solution curves of (1) on a two-dimensional center manifold $\mathcal{S}$ in phase space.

In figure 2, the vertical axis corresponds to the parameter $c=\sqrt{2 \rho D}\left(\frac{1}{2}-\alpha\right)$ and the horizontal axis correponds to the translation variable $a$. The dynamics on this two-dimensional center manifold $\mathcal{S}$ are quite simple, and can be described by the system of ordinary differential equations (ODEs) $\dot{a}=c, \dot{c}=0$. The line at $c=0$, which is labelled as $\Theta$ in figure 2, corresponds to the case where the Allee parameter $\alpha$ has a value of $1 / 2$, and propagation failure occurs. Any given point of this line $\Theta$ corresponds, in physical space, to a steady-state wave profile, let's say $u^{*}(x)$. Futhermore, different points on $\Theta$ correspond to translated versions (in space) of the same basic wave profile, i.e. $\left(\mathcal{T}_{a} u^{*}\right)(x)=u^{*}(x+a)$.

On the straight lines $c=c^{*} \neq 0$, solutions of $\dot{a}=c, \dot{c}=0$ evolve in time at constant speed $c^{*}$ : if at time $t=0$ the wave profile is described in physical space by the function $u^{*}(x)$, then at time $t>0$, the wave profile is described by the function $\left(\mathcal{T}_{c^{*} t} u^{*}\right)(x)=u^{*}\left(x+c^{*} t\right)$, i.e. the same basic wave profile $u^{*}$ propagates linearly in physical space at constant speed $c^{*}$. So figure 2 is a complete summary of how travelling wave solutions to (1) are organized in the phase space of the dynamical system, and also illustrates the role played by the translation symmetry of (1). 


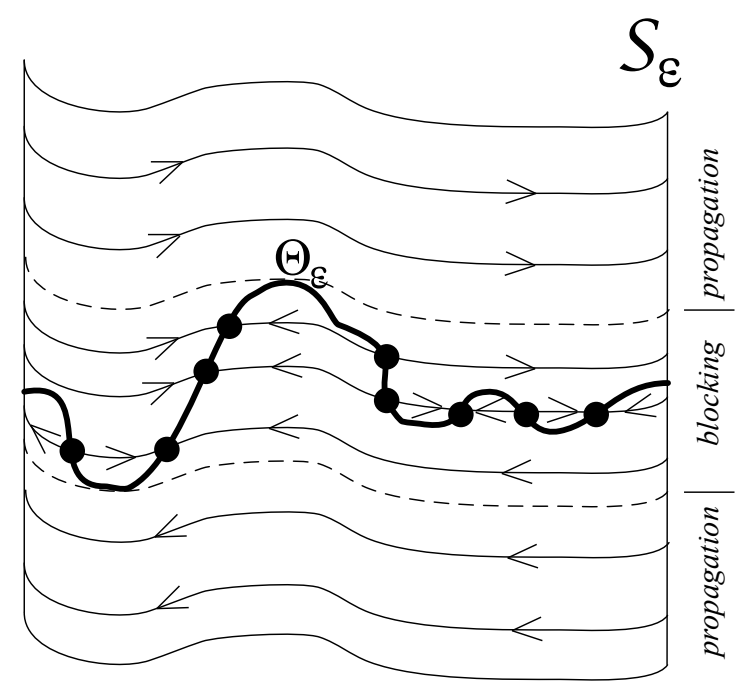

Figure 3: Flow of (6) on the perturbed center manifold $\mathcal{S}_{\varepsilon}$. All flow lines have constant value of $c$, but in contrast to figure 2, the quantity $\dot{a}$ is not constant (the wave propagation is not uniform in space). In the region between the dashed lines, equilibrium points on $\Theta_{\varepsilon}$ intersect flow lines, and thus lead to propagation failure or wave blocking. Outside this region, the heterogeneity is not large enough to impede propagation.

Now, suppose (1) is slightly perturbed to the following

$$
\frac{\partial u}{\partial t}=D \frac{\partial^{2} u}{\partial x^{2}}+\mathcal{F}(u)+\varepsilon \mathcal{G}(u, x),
$$

where $\mathcal{G}(u, x)$ could be a perturbation of the reaction term, or a perturbation to the diffusion coefficient, or a combination of both. The important thing is that $\mathcal{G}$ is assumed to depend non-trivially on the space variable $x$. Ecologically, $\mathcal{G}$ can represent spatial heterogeneity in population growth rate and/or diffusion, and if $\mathcal{G}$ is bounded in space, the small parameter $\varepsilon$ gives a measure of the size of the heterogeneous pertubation.

From a symmetry perspective, (6) no longer possesses the SE(1) translation symmetry described above, unless $\varepsilon=0$. We say that (6) undergoes forced symmetry-breaking when $\varepsilon \neq 0$. However, for $\varepsilon \neq 0$ small enough, we expect that the dynamics of (6) will be close to the dynamics of (1). In particular, the invariant manifold $\mathcal{S}$ for (1) should persist as an invariant manifold $\mathcal{S}_{\varepsilon}$ for (6), but the internal dynamics on $\mathcal{S}_{\varepsilon}$ will be perturbed as depicted in figure 3 .

On the perturbed center manifold $\mathcal{S}_{\varepsilon}$, the two-dimensional flow is described by the perturbed ODEs (to leading order in $\varepsilon$ ): $\dot{a}=c-\varepsilon r(a), \dot{c}=0$. The function $r(a)$ depends on $\mathcal{G}$ and other quantities, as we will see in the next subsection. The line of equilibria $\Theta$ thus gets perturbed to a curve of equilibria $\Theta_{\varepsilon}$ represented locally (for small enough $\varepsilon$ ) as $c=\varepsilon r(a)$. In figure 3 , we 
see that there is a range of $c$ values (between the two dashed lines) of width approximately $\varepsilon\left(\sup _{a \in \mathbb{R}} r(a)-\inf _{a \in \mathbb{R}} r(a)\right)$ in which the flow lines $c=$ constant meet equilibrium points on $\Theta_{\varepsilon}$. These equilibria correspond to wave profiles which are steady in physical space, and thus fail to propagate. Outside of this region, waves can propagate unimpeded (the heterogeneity is not of sufficient strength or size to block the propagation). In contrast to travelling waves for (1), the speed of travelling is not constant in space.

In the $(\varepsilon, c)$ parameter space of (6) there is thus a cone delimited by the straight lines $c=\left(\sup _{a \in \mathbb{R}} r(a)\right) \varepsilon$ and $c=\left(\inf _{a \in \mathbb{R}} r(a)\right) \varepsilon$ which approximately (to leading order in $\varepsilon$ ) determines the region in which propagation failure occurs in (6) (see Figure 4). In the next subsection, we present the technical details which make the above qualitative and heuristic arguments rigorous.

\subsection{Theoretical analysis}

To cast our problem in the framework of LeBlanc and Roy (2013), we formulate equation (1) as a dynamical system

$$
\frac{d u}{d t}=\mathcal{A} u+\mathcal{F}(u, \alpha),
$$

on the Banach space $Y=C_{\text {unif }}^{0}(\mathbb{R}, \mathbb{R})$, where $\mathcal{A}=\frac{\partial^{2}}{\partial x^{2}}$ denotes the diffusion operator. We explicitly include the dependence of the reaction term on a parameter $\alpha$. We make the following assumptions on the growth term.

Hypothesis 2.1 The function $\mathcal{F}(u, \alpha)$ is smooth and vanishes at the points $u_{-}$, $u_{0}$ and $u_{+}, u_{-}<u_{0}<u_{+}$, and only at these points. Furthermore, we assume $\mathcal{F}_{u}\left(u_{ \pm}, \alpha\right)<0$ and that $\mathcal{F}_{u}\left(u_{0}, \alpha\right)>0$.

In an ecological setting, the simplest form of the Hypothesis means that we have a strong Allee effect in the population dynamics: the steady states $u_{-}$and $u_{+}$ are locally stable while there is one intermediate unstable state, $u_{0}$.

Under these assumptions equation (7) has a unique (up to translation) traveling wave solution $u(x, t)=u^{*}(x+c t)$ with $\lim _{x \rightarrow \pm \infty} u^{*}(x)=u_{ \pm}$and there is a unique speed $c \in \mathbb{R}$ (Fife and McLeod, 1977; Volpert, Volpert, and Volpert, 1994). It is easy to see that the cubic nonlinearity (2) satisfies Hypothesis 2.1 with $u_{-}=0, u_{0}=\alpha$ and $u_{+}=1$. In this case, the unique speed $c$ is given by (3) and the traveling wave profile is as in (4).

For every $a \in \mathbb{R}$, we now define the translation operator $\mathcal{T}_{a}$ on $Y$ by $\mathcal{T}_{a} v(x)=$ $v(x+a)$ for any $v \in Y$. These translations define an action of the additive group of real numbers, $\mathrm{SE}(1)$, on $Y$. Equation (7) is said to be equivariant under this action since solutions of (7) are mapped to other solutions by this action. In the special case of the traveling wave solution, $u^{*}$, the time-orbit of the RDE is contained in the $\mathrm{SE}(1)$-orbit of the group action. Such a solution is called a relative equilibrium (Sandstede, Scheel, and Wulff, 1997).

The infinitesimal generator of the translation $\mathcal{T}: a \mapsto \mathcal{T}_{a}$ is given by the derivative 


$$
\xi u=\lim _{h \rightarrow 0} \frac{\mathcal{T}_{h} u(x)-u(x)}{h}=\frac{\partial u}{\partial x} .
$$

The operators $\mathcal{A}$ and $\xi$ are defined on dense subspaces of $Y$, and they commute with $\mathcal{T}_{a}$ and with each other, i.e $\mathcal{A} \xi=\xi \mathcal{A}, \xi \mathcal{T}_{a}=\mathcal{T}_{a} \xi$ and $\mathcal{A} \mathcal{T}_{a}=\mathcal{T}_{a} \mathcal{A}$ on appropriate dense subspaces of $Y$.

Now we introduce into (7) a perturbation that represents spatial heterogeneity and write

$$
\frac{d u}{d t}=\mathcal{A} u+\mathcal{F}(u, \alpha)+\varepsilon \mathcal{G}(\xi u, u, x ; \varepsilon),
$$

where $0<\varepsilon \ll 1$ is a small parameter.

Hypothesis 2.2 We make the following assumptions on the functions $\mathcal{F}$ and $\mathcal{G}:$

1. $\mathcal{F}$ is $S E(1)$-equivariant, that is $\mathcal{F}\left(\mathcal{T}_{a} u, \alpha\right)=\mathcal{T}_{a} \mathcal{F}(u, \alpha)$.

2. The function $\mathcal{G}$ is smooth, bounded and not $S E(1)$-equivariant. That is, there exists $a \in \mathbb{R}$ such that

$$
\mathcal{G}\left(\xi \mathcal{T}_{a} u, \mathcal{T}_{a} u, x ; \varepsilon\right) \neq \mathcal{T}_{a} \mathcal{G}(\xi u, u, x ; \varepsilon) .
$$

The ecological equivalents of this hypothesis are that, in the absence of perturbations, the system is spatially homogeneous, but the perturbation introduces spatial heterogeneity.

We are interested in characterizing the effects of the spatial heterogeneity $\mathcal{G}$ on the constant-speed traveling wave that exists when $\varepsilon=0$. Note that the fundamental difference between (10) and the perturbations that were studied by LeBlanc and Roy (2013) is that in (10), we allow the perturbation to also depend on the derivative term $\xi u$. We will need this generalization for the applications we study in the next section.

Proceeding as in LeBlanc and Roy (2013), we will assume that the relationship between the speed $c$ of the travelling wave solution $u(x, t)=u^{*}(x+c t)$ of $(7)$ and the parameter $\alpha$ defines a locally (around $c=0$ ) smooth function $\alpha(c)$. We then rewrite (7) as

$$
\begin{aligned}
& u_{t}=\mathcal{A} u+\mathcal{F}(u, \alpha(c)), \\
& c_{t}=0,
\end{aligned}
$$

on $Y \times \mathbb{R}$, and the relative equilibrium (travelling wave) is a solution of the form

$$
\left(\begin{array}{c}
u(t) \\
c(t)
\end{array}\right)=\left(\begin{array}{c}
\mathcal{T}_{a(t)} u^{*} \\
c
\end{array}\right),
$$

with $a(t)=c t$. This relation describes the situation in Figure 2 .

If we assume that the travelling wave profile $u^{*}$ is independent of $c$ (e.g. as in (4)), then a straightforward computation (LeBlanc and Roy, 2013; Roy, 
2013) shows that the linearization of (11) at the relative equilibrium gives the linear operator $L: Y \times \mathbb{R} \rightarrow Y \times \mathbb{R}$ defined by

$$
L\left(\begin{array}{c}
\phi \\
w
\end{array}\right)=\left(\begin{array}{c}
\mathcal{A} \phi+\mathcal{F}_{u}\left(u^{*}, \alpha(0)\right) \phi+\xi u^{*} w \\
0
\end{array}\right) .
$$

Observe that

$$
L\left(\begin{array}{c}
\xi u^{*} \\
0
\end{array}\right)=\left(\begin{array}{l}
0 \\
0
\end{array}\right) \quad \text { and } \quad L\left(\begin{array}{l}
0 \\
1
\end{array}\right)=\left(\begin{array}{c}
\xi u^{*} \\
0
\end{array}\right),
$$

i.e, $L$ has a zero eigenvalue with (generalized) eigenvectors given by $\phi_{1}=$ $\left(\xi u^{*}, 0\right)^{T}$ and $\phi_{2}=(0,1)^{T}$. Thus, the corresponding eigenspace, $E=\operatorname{span}\left\{\phi_{1}, \phi_{2}\right\}$, is two dimensional. We will assume the following

Hypothesis 2.3 (Spectral Gap Condition ((LeBlanc and Roy, 2013), Hypothesis 3.3a, $p$ 13)) Assume that the zero eigenvalue of $L$ is such that the corresponding generalized eigenspace $E$ is two dimensional, with spectral projection $P$. Furthermore, we will assume that all other elements of the spectrum of $L$ are bounded away from the imaginary axis in the left plane.

We note that for the cubic nonlinearity (2), the operator $L$ satisfies hypothesis 2.3 (LeBlanc and Roy, 2013; Roy, 2013). We are not aware of any simple ecological interpretation or equivalent of this hypothesis. However, we point out that this hypothesis is violated in the case of so-called "pulled waves" that connect an unstable to a stable equilibrium. In those cases, there typically is an entire range of speeds for which a travelling wave exists, and there is no spectral gap. The following hypothesis is of a technical nature, required for the proofs rather than for ecological applications.

Hypothesis 2.4 We will assume that

1. The $\operatorname{map} a \rightarrow \mathcal{T}_{a} u^{*}$ is $C^{k+2}$.

2. The map $a \rightarrow \mathcal{T}_{a} v$ is $C^{k+2}$ whenever $v \in E$.

3. For all $K>0, \exists \delta>0$ such that $\left\|\mathcal{T}_{a} u^{*}-u^{*}\right\| \geq \delta$ whenever $|a|>K$.

4. The projections $\mathcal{T}_{a} P \mathcal{T}_{-a}$ are $C^{k+1}$ in $a \in \mathrm{SE}(1)$ in the operator norm.

It follows from LeBlanc and Roy (2013) that the projection operator is

$$
P\left(\begin{array}{c}
v(x) \\
c
\end{array}\right)=\frac{\left\langle\phi_{1},\left(\begin{array}{c}
v(x) \\
c
\end{array}\right)\right\rangle}{\left\langle\phi_{1}, \phi_{1}\right\rangle} \phi_{1}+\frac{\left\langle\phi_{2},\left(\begin{array}{c}
v(x) \\
c
\end{array}\right)\right\rangle}{\left\langle\phi_{2}, \phi_{2}\right\rangle} \phi_{2},
$$

and defines an $L$-invariant splitting of the space $Y \times \mathbb{R}=\operatorname{range}(P) \oplus \operatorname{ker}(P)=$ $E \oplus W$, with the scalar product defined as

$$
\left\langle\left(\begin{array}{c}
u(x) \\
a
\end{array}\right),\left(\begin{array}{c}
v(x) \\
b
\end{array}\right)\right\rangle=\int_{\mathbb{R}} u(x) v(x) d x+a b .
$$


We note that the cubic nonlinearity $(2)$ and the projection operator $P$ satisfy hypothesis 2.4 (LeBlanc and Roy, 2013; Roy, 2013). Applying the center manifold theorem of Sandstede, Scheel, and Wulff (1997) and suitably extending the proofs by LeBlanc and Roy (2013), we get the following main result whose detailed proof is in the Appendix.

Theorem 2.5 Consider the nonlinear differential equation

$$
u_{t}=\mathcal{A} u+\mathcal{F}(u, \alpha(c))+\varepsilon \mathcal{G}(\xi u, u, x ; \varepsilon)
$$

which generates a local semi flow on a Banach space $Y$. Suppose that $\mathcal{A}$ and $\xi$ are linear operators whose domains are dense in $Y$ and are such that there is an SE(1)-invariant subspace on which all operators commute. Furthermore, suppose that hypotheses 2.1-2.4 are satisfied. Then, for all $0 \leq \varepsilon \ll 1$ and all c near 0 , the semiflow admits a smooth stable invariant manifold $\mathcal{S}_{\varepsilon}$, on which the dynamics reduce to the ordinary differential equations

$$
\begin{aligned}
& a_{t}=c-\varepsilon r(a)+\varepsilon q(a, c, \varepsilon), \\
& c_{t}=0,
\end{aligned}
$$

where $q(a, c, \varepsilon)$ is a smooth function such that $q \rightarrow 0$ uniformly as $(\varepsilon, c) \rightarrow(0,0)$ and

$$
r(a)=-\frac{1}{\left\langle\phi_{1}, \phi_{1}\right\rangle} \int_{-\infty}^{\infty} \mathcal{T}_{-a} \mathcal{G}\left(\xi \mathcal{T}_{a} u^{*}, \mathcal{T}_{a} u^{*}, x, 0\right) \cdot \xi u^{*} d x .
$$

The system in (18) generates the dynamics on the center manifold, as illustrated in Figure 3. Solutions of (17) that belong to $\mathcal{S}_{\varepsilon}$ correspond to perturbed relative equilibria, i.e. travelling waves with non-constant speed. Wave blocking or propagation failure corresponds to a steady-state on the center manifold, i.e. it occurs when $a_{t}=0$. The first two terms in the first equation in (18) then give a relationship (to first order in $\varepsilon$ ) between $c$ and $\varepsilon$ for wave blocking to occur. The largest and the smallest values of $c$ for wave blocking are hence given by the largest and smallest values of $r(a)$, respectively. Hence, wave blocking occurs in a cone with vertex at $(0,0)$ in $(c, \varepsilon)$ parameter space, bounded by curves of the form

$$
c_{*}=\inf _{a \in \mathbb{R}}\{r(a)\} \varepsilon+O\left(\varepsilon^{2}\right) \text { and } c^{*}=\sup _{a \in \mathbb{R}}\{r(a)\} \varepsilon+O\left(\varepsilon^{2}\right) .
$$

The cone is visualized in Figure 4. In the next section, we will compute the infimum and supremum of the function $r(a)$ for several different applications. 


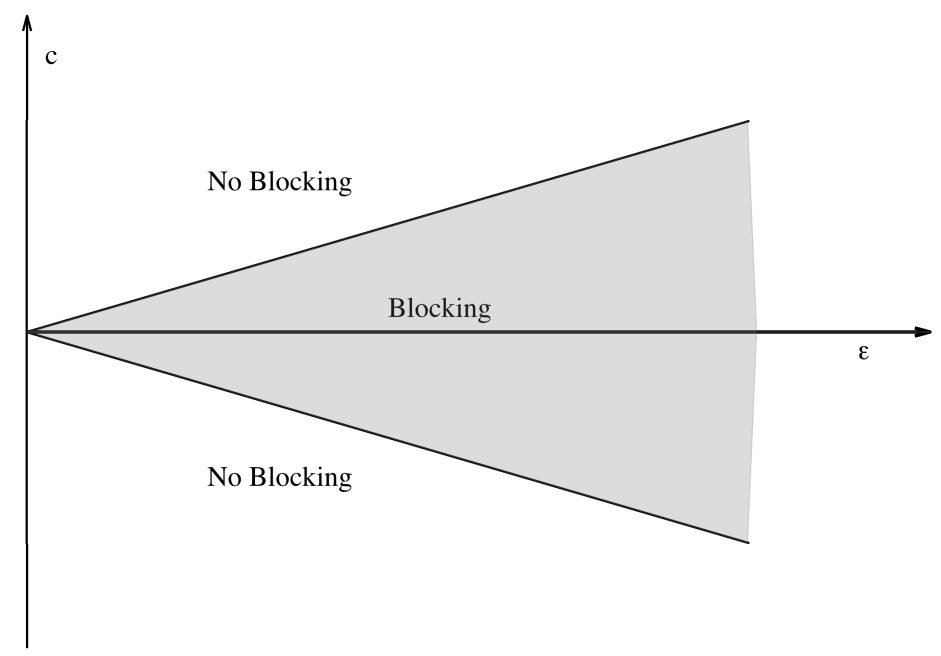

Figure 4: The cone of parameter values in $(c, \varepsilon)$ parameter space that leads to propagation failure. The slopes of the upper and lower bounds for the cone are given by the infimum and supremum of the function $r(a)$ as in the expressions in $(20)$.

\section{Applications}

In this section, we apply our results from Theorem 2.5 to find conditions under which periodic spatial variation can result in invasion pinning. In a homogeneous landscape, the dynamics of the species' density is governed by the equation

$$
\frac{\partial u}{\partial t}=D \frac{\partial^{2} u}{\partial x^{2}}+\rho u(1-u)(u-\alpha) .
$$

We consider various scenarios in which spatial heterogeneity affects only the population growth rate or only the movement term or both terms simultaneously. Theorem 2.5 provides a relationship between the speed parameter $c$ and the perturbation parameter $\varepsilon$. For applications, we consider parameters $\alpha$ and $\varepsilon$ instead since $\alpha$ is one of the actual model parameters and is, at least theoretically, measurable in the field.

According to Theorem 2.5 the maximum and minimum of the function $r(a)$ in (19) give the slope of the cone in $(c, \varepsilon)$ space. The denominator of this expression can be evaluated analytically, using the explicit form of $u^{*}$.

$$
\begin{aligned}
\left\langle\phi_{1}, \phi_{1}\right\rangle & =\int_{\mathbb{R}}\left(\xi u^{*}\right)^{2} d x \\
& =\int_{\mathbb{R}}\left(\frac{\sqrt{2}}{8} \operatorname{sech}^{2}\left(\frac{\sqrt{2}}{4} x\right)\right)^{2} d x=\frac{\sqrt{2}}{12} .
\end{aligned}
$$


The numerator in (19) has to be evaluated numerically. We can do this efficiently by using the fact that $\mathcal{G}$ and $u^{*}$ are bounded and $\xi u^{*}$ is localized. Furthermore, due to the periodic nature of the environment $r(a)$ is also periodic. We used Simpson's rule on an appropriate interval to determine the corresponding value of $r(a)$.

We illustrate the validity of our theorem by numerical simulation of the corresponding reaction-diffusion equation. We choose a translation of $u^{*}$ as the initial condition. We then search, for each fixed value of $\alpha$, the smallest value of $\varepsilon$ for which the wave fails to advance; see also Figure 1. Failure to advance is determined by comparing the difference between $u(0, x)$ and $u\left(t_{f}, x\right)$, for sufficiently large $t_{f}$. The values determined through this procedure are compared with the corresponding approximation from the theorem.

\section{Perturbed Growth Term}

We begin with the assumption that only the growth term varies spatially. We choose $\rho=\rho(x)=1+\varepsilon \sin (x)$ and set $D=1$. We can rewrite (21) in the form (17) with

$$
\mathcal{G}(\xi u, u, x ; \varepsilon)=\sin (x) u(1-u)(u-\alpha) .
$$

The spatial period of the perturbation here and in the following examples is $L=2 \pi$. Figure 5 shows a graph of the corresponding function $r(a)$ in this case. Numerically, we find the values $r_{\max }=\max \{r(a)\} \approx 0.1567$ and $r_{\min }=-r_{\max }$. Thus, we anticipate that the invasion will fail whenever $|c / \varepsilon|<0.1567$. The relation $c=\sqrt{2}\left(\frac{1}{2}-\alpha\right)$ allows us to write the conditions for invasion pinning as

$$
\frac{1}{2}-\frac{r_{\min }}{\sqrt{2}} \varepsilon>\alpha>\frac{1}{2}-\frac{r_{\max }}{\sqrt{2}} \varepsilon .
$$

The illustration in Figure 6 shows an excellent agreement between the theoretically and numerically obtained values. 


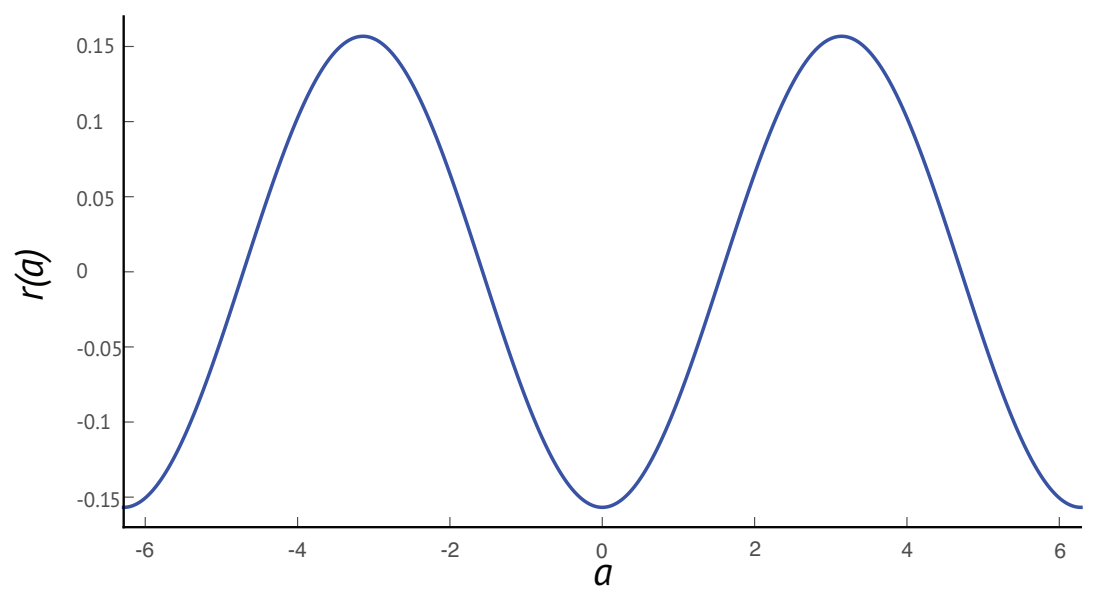

Figure 5: Plot of $r(a)$ for $\mathcal{G}$ given by $(22)$

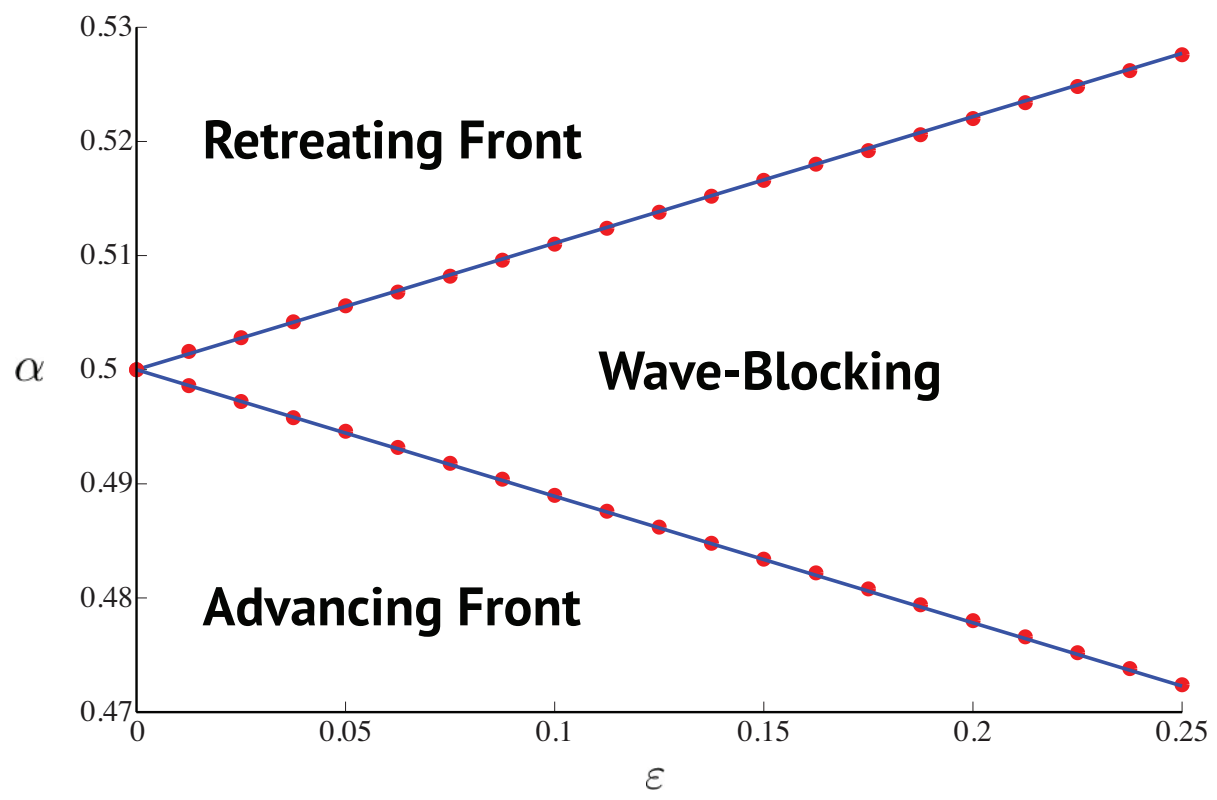

Figure 6: Comparison between the theoretical region of invasion pinning (blue lines) given by theorem 2.5 and the observed blocking region (red circles) for the perturbed growth function in (22). The approximation remains highly accurate for $\varepsilon<0.25$. 


\section{Perturbed Diffusion Term I}

When individuals can sense their environment, one expects that they will adjust their movement behavior to local conditions. There are several ways to formulate spatially varying diffusion models. We begin with so-called 'ecological diffusion', which can be derived from an individual-movement model. The equation reads

$$
\frac{\partial u}{\partial t}=\frac{\partial^{2}}{\partial x^{2}}[\mu(x) u]+u(1-u)(u-\alpha),
$$

where $\mu(x)$ denotes the motility of individuals (Turchin, 1998). As before, we consider a sinusoidal perturbation and write $\mu(x)=1-\varepsilon \sin (x)$.

Expanding the diffusion term

$$
\frac{\partial^{2}}{\partial x^{2}}[\mu(x) u]=\mu \frac{\partial^{2}}{\partial x^{2}} u+2 \mu^{\prime} \frac{\partial}{\partial x} u+\mu^{\prime \prime} u,
$$

we note that (24) is not of the form (21). We introduce the change of variables $x=y+\varepsilon \zeta(y)$ to transform the coefficient of the second-derivative term of $u$ to unity. To that end, we choose the function $\zeta(y)$ such that it satisfies the differential equation

$$
1-\varepsilon \sin (y+\varepsilon \zeta(y))=\left(1+\varepsilon \zeta^{\prime}(y)\right)^{2} .
$$

and such that $\varepsilon \zeta(y) \rightarrow 0$ as $\varepsilon \rightarrow 0$. This change of variables transforms (24) into the equation

$$
u_{t}=u_{y y}+u(1-u)(u-\alpha)+\varepsilon g\left(u_{y}, u, y, \varepsilon\right),
$$

where

$$
\begin{aligned}
& g\left(u_{y}, u, y, \varepsilon\right)= \\
& \sin (y+\varepsilon \zeta(y)) u-\left[\frac{2 \cos (y+\varepsilon \zeta(y))}{1+\varepsilon \zeta^{\prime}(y)}+\frac{\zeta^{\prime \prime}(y)(1-\varepsilon \sin (y+\varepsilon \zeta(y))}{\left(1+\varepsilon \zeta^{\prime}(y)\right)^{3}}\right] \frac{\partial u}{\partial y} .
\end{aligned}
$$

In this form, we can apply our theorem and calculate the maximum and minimum of the function $r(a)$. Using the identities

$$
\begin{aligned}
y+\left.\varepsilon \zeta(y)\right|_{\varepsilon=0} & =y, \\
1+\left.\varepsilon \zeta^{\prime}(y)\right|_{\varepsilon=0} & =1, \\
\left.\zeta^{\prime \prime}(y)\right|_{\varepsilon=0} & =-\frac{1}{2} \cos (y)
\end{aligned}
$$

we get

$$
r(a)=-\frac{12}{\sqrt{2}} \int_{-\infty}^{\infty}\left(\sin (y-a) u^{*}-\frac{3}{2} \cos (y-a) \xi u^{*}\right) \cdot \xi u^{*} d y .
$$

Integrating numerically, we find that $\max \{r(a)\}=\min \{r(a)\} \approx 0.47037$. So, we expect invasion pinning to occur for values of $\alpha$ defined by the inequality 
(23). Numerical simulations confirm that this approximation is valid as $\varepsilon \rightarrow 0$. As $\varepsilon$ increases, the lower value of $\alpha$ is still captured very well, but the upper value is not; see Figure 7.

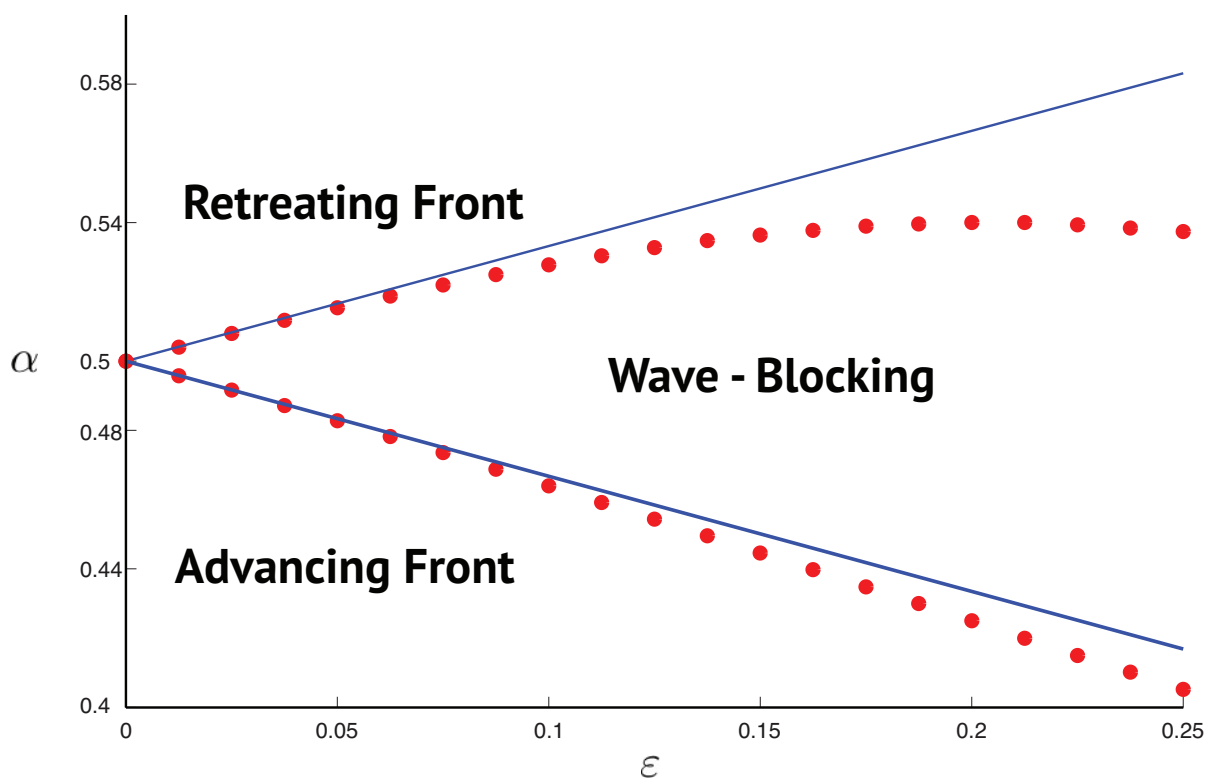

Figure 7: Comparison between the theoretical region of invasion pinning (blue lines) given by theorem 2.5 and the to observed blocking region (red circles) for the ecological diffusion model (24) with $\mu(x)=1-\varepsilon \sin (x)$.

\section{Perturbed Diffusion Term II}

An alternative derivation of the diffusion equation starts from a conservation law and makes the assumption of Fickian flux to relate flux to density. The resulting RDE becomes

$$
\frac{\partial u}{\partial t}=\frac{\partial}{\partial x}\left[D(x) \frac{\partial u}{\partial x}\right]+u(1-u)(u-\alpha)
$$

where $D(x)$ represents the spatially varying diffusion coefficient. For the purposes of this application we will again choose $D(x)=1-\varepsilon \sin (x)$.

As before, equation (26) is not in the form (21), but also as before, a suitable change of coordinates $x=y+\varepsilon \zeta(y)$ will render equation (26) into the form

$$
u_{t}=u_{y y}+u(1-u)(u-\alpha)+\varepsilon g\left(u_{y}, u, y, \varepsilon\right),
$$

where now

$$
g\left(u_{y}, u, y, \varepsilon\right)=\left(-\frac{\zeta^{\prime \prime}(1-\varepsilon \sin (y+\varepsilon \zeta(y)))}{\left(1+\varepsilon \zeta^{\prime}\right)^{3}}-\frac{\cos (y+\varepsilon \zeta(y))}{1+\varepsilon \zeta^{\prime}}\right) \frac{\partial u}{\partial y}
$$


As before, we numerically evaluate the required function

$$
r(a)=-\frac{6}{\sqrt{2}} \int_{-\infty}^{\infty}\left[\cos (y-a) \xi u^{*}\right] \cdot \xi u^{*} d y .
$$

We find the values $\max \{r(a)\}=0.15679$ and $\min \{r(a)\}=-0.15679$. The comparison between theoretical and simulated conditions for invasion pinning reveals a very good agreement for the entire range of $\varepsilon$; see Figure 8.

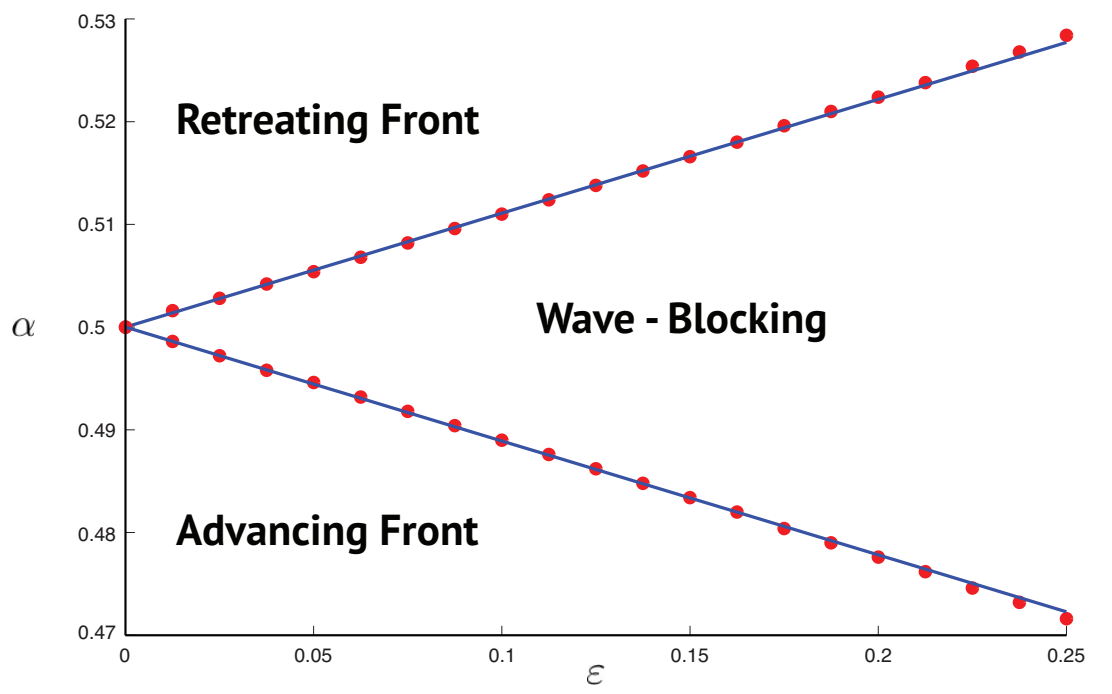

Figure 8: Comparison between the theoretical region of invasion pinning (blue lines) given by theorem 2.5 and the observed blocking region (red circles) for the Fickian diffusion model (26) with $D(x)=1-\varepsilon \sin (x)$.

Comparing Figures 7 and 8, we note that the slope of the blocking wedge with ecological diffusion is much larger (threefold) than with Fickian diffusion. We also note that the approximation given by theorem 2.5 is valid for a larger range of $\varepsilon$ with Fickian diffusion, but that the range of $\alpha$ for which the approximation is good is almost the same for the two different movement operators. The two descriptions of movement are, of course, related. We can write

$$
\frac{\partial}{\partial x}\left[D(x) \frac{\partial u}{\partial x}\right]=\frac{\partial^{2}}{\partial x^{2}}[D(x) u]-\frac{\partial}{\partial x}\left[D^{\prime}(x) u\right] .
$$

We see that Fickian diffusion is the same as ecological diffusion with a movement bias in the direction of $D^{\prime}(x)$ : where $D^{\prime}<0$ the bias is to the left; where $D^{\prime}>0$ the bias is to the right. This spatially periodic bias towards regions where $D(x)$ is high is what makes the invasion-pinning wedge in parameter space so much smaller. We explore this question in the context of spatially varying resource abundance in more detail in the following section. 


\section{Spatial Correlation}

Spatial heterogeneity is likely to affect both, reproductive ability and dispersal behaviour. In fact, foraging theory explains and explores how dispersal behaviour should depend on habitat quality. To explore how different dispersal strategies affect the invasion-pinning wedge, we combine the spatially varying growth rate from (22) with each of the movement models from (24) and (26). More precisely, we assume that diffusion coefficient and motility depend on the deviation of the growth rate from its average. We set

$$
\begin{aligned}
\rho(x) & =1-\varepsilon \sin (x), & \\
D(x)=\mu(x) & =1+\delta(\rho-\langle\rho\rangle)=1-\varepsilon \delta \sin (x), & |\varepsilon \delta|<1,
\end{aligned}
$$

where $\langle\rho\rangle$ is the spatial average of $\rho$. Note that the sign of $\delta$ is the correlation between $\rho(x)$ and $D(x)$ or $\mu(x)$. When $\delta>0$, then diffusion and motility are high where the growth rate is high; when $\delta<0$ then diffusion and motility are low where the growth rate is high.

We use the same change of variables as before to transform the equations to equations of the form (17). Applying our theorem and numerically integrating as we have done in the previous sections, we plot the maximum of $r(a)$ as a function of $\delta$. The results are illustrated in Figure 9.
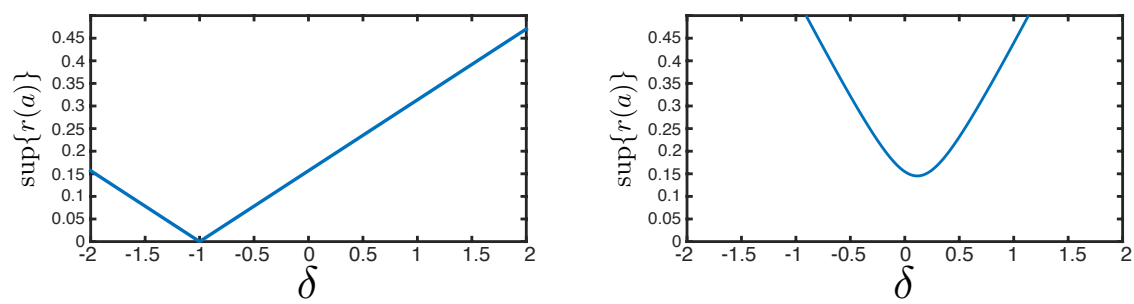

Figure 9: Plot of the slope of the invasion-pinning wedge $\max \{r(a)\}$ as a function of $\delta$. The plot on left corresponds to the Fickian flux equation (26); the plot on the right represents ecological diffusion in (24). In both cases, we use growth rate $\rho(x)$ as in (27) and diffusivity and motility as in (28).

Even though both curves show a unique minimum, there are several salient differences between the two cases. For Fickian diffusion, the slope of the invasionpinning wedge has a minimum very close to $\delta=-1$, i.e. when the growth rate and diffusivity are opposites. The minimum is zero so that there is essentially no invasion-pinning for small values of $\varepsilon$. By contrast, with ecological diffusion, the minimum value of $\max \{r(a)\}$ occurs for small but positive $\delta$, i.e. when growth and motility are in complete correlation but motility has only a small amplitude. The minimum value of $\max \{r(a)\}$ is positive, indicating that invasion-pinning occurs even for very small $\varepsilon$.

We aim to interpret this result from a mechanistic point of view. When $\delta<0$, then regions of high motility correspond to regions of low growth rate; see Figure 
10. In the absence of an Allee effect, this behaviour is beneficial since individuals transverse 'bad' areas quickly and move slowly in 'good' areas (Maciel and Lutscher, 2013). In the presence of an Allee effect, however, the situation is more subtle. High motility in low-growth areas leads to low population densities there, which results in higher population loss-rates due to the Allee effect. Therefore, an invasion with ecological diffusion can get pinned more easily when $\delta<0$. On the other hand, low motility in high-growth areas concentrates individuals there so that a population that might retreat in a homogeneous landscape can persist in the high-growth rate patches and does not retreat in the heterogeneous environment.

Fickian diffusion, as we saw above, can be interpreted as motility with bias towards high-motility regions. If $\delta<0$, then this bias towards high-motility areas may seem counter-productive since it concentrates individuals in regions of low growth rates. In the absence of an Allee effect, such a strategy is expected to be detrimental. In the presence of an Allee effect, however, this bias towards lowgrowth regions increases the density in low-growth regions and thereby decreases the loss-rate there. The population continues to invade even when the spatial perturbation is relatively large. When the bias towards low-growth regions is too strong, i.e. $\delta<-1$, then the bias away from high-growth regions is detrimental and leads to invasion-pinning. If $\delta>0$ then the movement bias is towards highgrowth regions. The resulting concentration of individuals there allows for local population persistence but not for spread. Hence, an invasion may get pinned more easily and a retreating population may be halted.

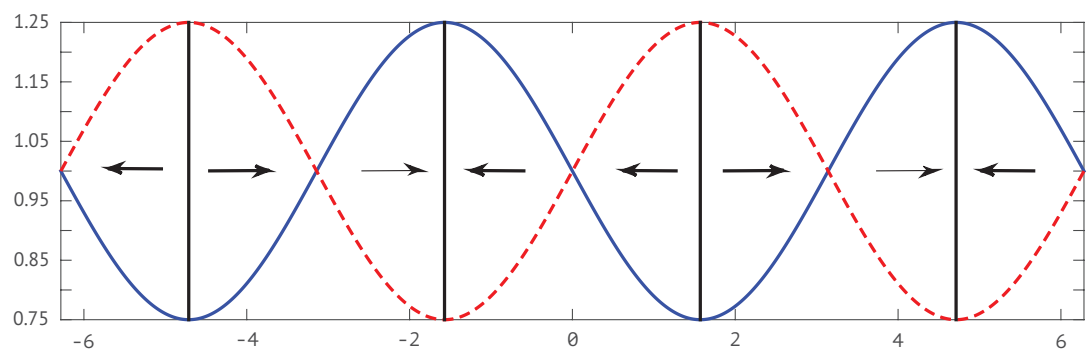

Figure 10: Plot of $D$ (dashed red line) and $\rho$ (solid blue line) for $\varepsilon=0.25$ and $\delta=-1$. The arrows indicate the direction of the movement bias.

\section{Conclusion}

Biological invasions and their concomitant environmental and economic damage are a massive challenge for science, policy and management. Spatial heterogeneity in particular affects so many aspects of an invasion process that its overall effect on the rate of propagation is hard to predict. Our work sheds a new light on one particular aspect of these processes, namely on how heterogeneity in movement and growth may pin an invasion of a population that exhibits 
an Allee effect. Our approach brings recent tools from equivariant bifurcation theory to bear on questions in spatial ecology.

Our work is based on a symmetry-breaking perturbation technique that was developed by LeBlanc and Roy (2013) and that we extend here to include spatial heterogeneity in the movement term. This extension is crucial for applications in ecology as many individuals adjust their movement behaviour to local, spatially varying conditions. However, the estimates in our theorem allow the perturbation to only include first derivative terms and not the naturally occurring second derivative. We used a change of variables to circumvent this difficulty. The technique that we used is not limited to scalar equations; in fact, it was formulated for vector-valued systems originally (LeBlanc and Roy, 2013). We could therefore extend the model to a stage-structured population and study the effects of component Allee effects (Courchamp, Berec, and Gascoigne, 2008) and stage-specific dispersal behaviour on invasion pinning. While abstract results on the existence and uniqueness of the travelling wave profile exist (Volpert, Volpert, and Volpert, 1994), there is usually no explicit formula as we have in (4). To compute the slope of the invasion-pinning wedge, one would first solve the homogeneous problem to obtain the shape of the travelling wave and then evaluate the extreme values of the function $r(a)$ numerically. What is needed, however, is the spectral gap of the operator (see hypothesis 2.3) that leads to the uniqueness of the wave speed and profile. In other words, the technique used here does not apply to so-called monostable waves where there is no Allee effect.

Our approach is complementary to the work by Maciel and Lutscher (2015). There, the authors used homogenization, i.e. the spatial heterogeneity had to be on a small scale, but the effect of heterogeneity could be large. Here, we use perturbation so that the effect of heterogeneity, represented by parameter $\varepsilon$, has to be small but the spatial scale could be large. We see that the results hold in the limit as $\varepsilon \rightarrow 0$. While the range of $\varepsilon$ for which the approximation holds can sometimes be reasonably large (Figures 6 and 8), this is not always the case (Figure 7 ).

Our approach is specific to the growth function having an Allee effect and does not translate to the Fisher-KPP case because of the lack of a spectral gap, see Hypothesis 2.3. However, the Fisher-KPP case is much more amenable to standard analytical techniques because the spreading speed in that case is determined by the linearization at zero, and the linear equation can be treated more easily. While introducing spatial heterogeneity into an equation of either type (with or without Allee effect) can be seen as a small perturbation, introducing an Allee effect into a model without Allee effect is typically not a small perturbation and requires completely different techniques, one of which we present in this work.

The ecological insights from our work contain positive and negative aspects for managers of biological invasions. On the positive side, it may be possible to pin an invasion where the population experiences an Allee effect via landscape heterogeneity. Control measures do not have to be applied uniformly throughout a landscape. A treatment analogous to regularly spaced 'fire breaks' could be 
sufficient so stop an invasion, even when the perturbation is not particularly large. On the negative side, what helps pin a successful invasion might also pin a retreating wave. In other words, a potential invader that cannot persist and spread in a homogeneous environment might be able to stay present in a slightly heterogeneous habitat.

Our model considered habitat heterogeneity as a periodic variation in growth rate. Previous work on spread in the presence of an Allee effect (Berestycki, Bouhours and Chapuisat, 2016) focused on the geometry of a habitat. Those authors showed that a sudden increase in the width of a habitat could inhibit a population from spreading. We plan to investigate in the future how our symmetry-breaking approach can be applied to study the effects of habitat geometry on population propagation. Another future project will be to apply the ideas developed here to spread models other than reaction-diffusion equations, for example, integrodifferential and integrodifference equations.

\section{A Proof of Theorem 2.5}

We rewrite (17) as

$$
\begin{aligned}
& u_{t}=\mathcal{A} u+\mathcal{F}(u, \alpha)+\varepsilon \mathcal{G}(\xi u, u, x ; \varepsilon) \\
& c_{t}=0
\end{aligned}
$$

The existence of the two-dimensional center manifold $\mathcal{S}_{\varepsilon}$ follows from Sandstede, Scheel, and Wulff (1997) and LeBlanc and Roy (2013). To get the dynamics of (29) restricted to $\mathcal{S}_{\varepsilon}$, we follow (Sandstede, Scheel, and Wulff, 1997) and use the following parametrization of a local neighborhood of the relative equilibrium at $\varepsilon=0$ :

$$
\left(\begin{array}{l}
u \\
c
\end{array}\right)=\mathcal{T}_{a}\left[\left(\begin{array}{c}
u^{*} \\
0
\end{array}\right)+c\left(\begin{array}{l}
0 \\
1
\end{array}\right)+\varepsilon\left(\begin{array}{l}
w \\
0
\end{array}\right)\right],
$$

where $w$ is a bounded uniformly continuous function with bounded and uniformly continuous derivative.

Following closely LeBlanc and Roy (2013), we substitute parameterization (30) into system (29), and obtain

$$
\begin{aligned}
\dot{a}(t) \mathcal{T}_{a(t)}\left(\left(\begin{array}{c}
\xi u^{*} \\
0
\end{array}\right)+O(\varepsilon)\right) & =\mathcal{T}_{a(t)}\left(\left(\begin{array}{c}
\mathcal{A} u^{*} \\
0
\end{array}\right)+\left(\begin{array}{c}
\varepsilon \mathcal{A} w \\
0
\end{array}\right)\right) \\
& +\mathcal{T}_{a(t)}\left(\begin{array}{c}
\mathcal{F}\left(u^{*}+\varepsilon w, c\right) \\
0
\end{array}\right) \\
& +\varepsilon\left(\begin{array}{c}
\mathcal{G}\left(\mathcal{T}_{a}\left(\xi u^{*}+\varepsilon \xi w\right), \mathcal{T}_{a}\left(u^{*}+\varepsilon w\right), x, \varepsilon\right) \\
0
\end{array}\right) .
\end{aligned}
$$

Applying $\mathcal{T}_{-a(t)}$ to both sides of the equation above gives 


$$
\begin{aligned}
\dot{a}(t)\left(\left(\begin{array}{c}
\xi u^{*} \\
0
\end{array}\right)+O(\varepsilon)\right) & =\left(\left(\begin{array}{c}
\mathcal{A} u^{*} \\
0
\end{array}\right)+\left(\begin{array}{c}
\varepsilon \mathcal{A} w \\
0
\end{array}\right)\right)+\left(\begin{array}{c}
\mathcal{F}\left(u^{*}+\varepsilon w, c\right) \\
0
\end{array}\right) \\
& +\varepsilon\left(\begin{array}{c}
\mathcal{T}_{-a(t)} \mathcal{G}\left(\mathcal{T}_{a}\left(\xi u^{*}+\varepsilon \xi w\right), \mathcal{T}_{a}\left(u^{*}+\varepsilon w\right), x, \varepsilon\right) \\
0
\end{array}\right) .
\end{aligned}
$$

Since $u^{*}$ is the wave profile for the unperturbed system know that

$$
\left(\begin{array}{c}
\mathcal{A} u^{*} \\
0
\end{array}\right)=-\left(\begin{array}{c}
\mathcal{F}\left(u^{*}, 0\right) \\
0
\end{array}\right)
$$

From the definition of the linear operator in (13), we have

$$
L\left(\begin{array}{l}
0 \\
c
\end{array}\right)=\left(\begin{array}{c}
\mathcal{F}_{c}\left(u^{*}, 0\right) c \\
0
\end{array}\right), \quad L\left(\begin{array}{c}
\varepsilon w \\
0
\end{array}\right)=\left(\begin{array}{c}
\varepsilon \mathcal{A} w+\varepsilon \mathcal{F}_{u}\left(u^{*}, 0\right) w \\
0
\end{array}\right),
$$

and so we write the previous expression as

$$
\begin{aligned}
\dot{a}(t)\left(\left(\begin{array}{c}
\xi u^{*} \\
0
\end{array}\right)+O(\varepsilon)\right) & =L\left(\begin{array}{l}
0 \\
c
\end{array}\right)+\varepsilon L\left(\begin{array}{c}
w \\
0
\end{array}\right)+\left(\begin{array}{c}
\mathcal{F}\left(u^{*}+\varepsilon w, c\right) \\
0
\end{array}\right) \\
& -\left(\begin{array}{c}
\mathcal{F}\left(u^{*}, 0\right) \\
0
\end{array}\right)-\left(\begin{array}{c}
\mathcal{F}_{c}\left(u^{*}, 0\right) c \\
0
\end{array}\right)-\varepsilon\left(\begin{array}{c}
\mathcal{F}_{u}\left(u^{*}, 0\right) w \\
0
\end{array}\right) \\
& +\varepsilon\left(\begin{array}{c}
\mathcal{T}_{-a(t)} \mathcal{G}\left(\mathcal{T}_{a(t)}\left(\xi u^{*}+\varepsilon \xi w\right), \mathcal{T}_{a(t)}\left(u^{*}+\varepsilon w\right), x, \varepsilon\right) \\
0
\end{array}\right) .
\end{aligned}
$$

Hypothesis 2.2, along with the fact that $w$ is a uniformly continuous function and bounded with respect to $\xi$, tells us that as $(\varepsilon, c) \rightarrow(0,0)$ the difference

$$
\mathcal{T}_{-a} \mathcal{G}\left(\mathcal{T}_{a}\left(\xi u^{*}+\varepsilon \xi w\right), \mathcal{T}_{a}\left(u^{*}+\varepsilon w\right), x, \varepsilon\right)-\mathcal{T}_{-a} \mathcal{G}\left(\mathcal{T}_{a}\left(\xi u^{*}\right), \mathcal{T}_{a} u^{*}, x, 0\right)
$$

tends to zero. Thus we introduce the function

$$
\begin{aligned}
q(a, c, \varepsilon) & =\frac{1}{\varepsilon}\left(\mathcal{F}\left(u^{*}+\varepsilon w, c\right)-\mathcal{F}\left(u^{*}, c\right)\right)-\mathcal{F}_{u}\left(u^{*}, c\right) w+\mathcal{F}_{u}\left(u^{*}, c\right) w-\mathcal{F}_{u}\left(u^{*}, 0\right) w \\
& +\mathcal{T}_{-a} \mathcal{G}\left(\mathcal{T}_{a}\left(\xi u^{*}+\varepsilon \xi w\right), \mathcal{T}_{a}\left(u^{*}+\varepsilon w\right), x, \varepsilon\right)-\mathcal{T}_{-a} \mathcal{G}\left(\mathcal{T}_{a}\left(\xi u^{*}\right), \mathcal{T}_{a} u^{*}, x, 0\right)
\end{aligned}
$$

where $q(a, c, \varepsilon) \rightarrow 0$ as $(\varepsilon, c) \rightarrow(0,0)$. Adding and subtracting $(\varepsilon q, 0)^{T}$ from the equation gives us

$$
\begin{aligned}
\dot{a}(t)\left(\left(\begin{array}{c}
\xi u^{*} \\
0
\end{array}\right)+O(\varepsilon)\right) & =L\left(\begin{array}{l}
0 \\
c
\end{array}\right)+\varepsilon L\left(\begin{array}{l}
w \\
0
\end{array}\right)+\varepsilon\left(\begin{array}{l}
q \\
0
\end{array}\right) \\
& +\varepsilon\left(\begin{array}{c}
\mathcal{T}_{-a} \mathcal{G}\left(\mathcal{T}_{a}\left(\xi u^{*}\right), \mathcal{T}_{a} u^{*}, x, 0\right) \\
0
\end{array}\right) .
\end{aligned}
$$


Applying the projection $P$ to the above equation and using the fact that $L(0, c)^{T}=$ $c\left(\xi u^{*}, 0\right)^{T}$ and $P\left(\xi u^{*}, 0\right)=\left(\xi u^{*}, 0\right)=\psi_{1}$ gives us

$$
\dot{a}\left(\psi_{1}+O(\varepsilon)\right)=c \psi_{1}+\varepsilon P\left(\begin{array}{c}
\mathcal{T}_{-a} \mathcal{G}\left(\xi \mathcal{T}_{a} u^{*}, \mathcal{T}_{a} u^{*}, x, 0\right) \\
0
\end{array}\right)+\varepsilon P\left(\begin{array}{l}
q \\
0
\end{array}\right)
$$

Projecting onto $\psi_{1}$ gives

$$
\begin{aligned}
\dot{a}\left(\left\langle\psi_{1}, \psi_{1}\right\rangle(1+O(\varepsilon))\right)=c\left\langle\psi_{1}, \psi_{1}\right\rangle & +\varepsilon\left\langle\left(\begin{array}{c}
\mathcal{T}_{-a(t)} \mathcal{G}\left(\xi \mathcal{T}_{a} u^{*}, \mathcal{T}_{a} u^{*}, x, 0\right) \\
0
\end{array}\right), \psi_{1}\right\rangle \\
& +\varepsilon\left\langle\left(\begin{array}{l}
q \\
0
\end{array}\right), \psi_{1}\right\rangle .
\end{aligned}
$$

Since $\varepsilon$ and $c$ are assumed to be small we can neglect the higher order terms that appear as a result of dividing by $1+O(\varepsilon)$. Letting

$$
\begin{gathered}
r(a)=-\frac{\left\langle\left(\begin{array}{c}
\mathcal{T}_{-a} \mathcal{G}\left(\xi \mathcal{T}_{a} u^{*}, \mathcal{T}_{a} u^{*}, x, 0\right) \\
0
\end{array}\right), \psi_{1}\right\rangle}{\left\langle\psi_{1}, \psi_{1}\right\rangle}, \\
\hat{q}=\frac{\left\langle\left(\begin{array}{l}
q \\
0
\end{array}\right), \psi_{1}\right\rangle}{\left\langle\psi_{1}, \psi_{1}\right\rangle},
\end{gathered}
$$

we get the system of differential equations

$$
\begin{aligned}
a_{t} & =c-\varepsilon r(a)+\varepsilon \hat{q}(a, c, \varepsilon), \\
c_{t} & =0,
\end{aligned}
$$

where $\hat{q}(a, c, \varepsilon) \rightarrow 0$ uniformly as $(\varepsilon, c) \rightarrow(0,0)$.

Now, an application of the implicit function theorem tells us that for $(\varepsilon, c)$ close enough to $(0,0)$ there exists a curve of equilibria such that $a_{t}=0$. This curve is the graph of the function $c=\varepsilon r(a)+\varepsilon \sigma(a, \varepsilon)$ where $\sigma$ is some bounded function with the property $\sigma(a, 0)=0$ (LeBlanc and Roy, 2013; Roy, 2013). Thus, it follows that if

$$
\inf _{a \in \mathbb{R}}\{r(a)+\sigma(a, \varepsilon)\}<\frac{c}{\varepsilon}<\sup _{a \in \mathbb{R}}\{r(a)+\sigma(a, \varepsilon)\}
$$

then at some point between $a \in \mathbb{R}$ we expect $a_{t}=0$. This means that at some point in time the travelling front will reach an equilibrium point at which the wave is stationary. In other words, propagation of the wave is blocked. We remark that for $(\varepsilon, c)$ close enough to $(0,0)$ the term $\sigma(a, \varepsilon)$ becomes negligible. Hence, close to $(0,0)$, the parameter region where we expect propagation failure is approximated by $\inf _{a \in \mathbb{R}}\{r(a)\}$ and $\sup _{a \in \mathbb{R}}\{r(a)\}$. 


\section{References}

W. Allee. Principles of Animal Ecology. Saunders Co., Philadelphia PA, 1949.

H. Berestycki, J. Bouhours, and G. Chapuisat. Front blocking and propagation in cylinders with varying cross section. Calc. Var. Partial Differential Equations, 55:Art. 44, 2016.

H. Berestycki, F. Hamel, and N. Nadirashvili. The speed of propagation for KPP type problems. I: Periodic framework. J. Eur. Math. Soc., 7:173-213, 2005.

F. Courchamp, L. Berec, and J. Gascoigne. Allee Effects in Ecology and Conservation. Oxford University Press, New York, 2008.

W. Ding, F. Hamel, and X.-Q Zhao. Transition fronts for periodic bistable reaction-diffusion equations. Calc. Var. Partial Differential Equations, $54: 2517-2551,2015$.

W. Ding, F. Hamel, and X.-Q Zhao. Bistable pulsating fronts for reactiondiffusion equations in a periodic habitat. Indiana Univ. Math. J., 66:11891265, 2017.

P. C. Fife and J. B. McLeod. The approach of solutions of nonlinear diffusion equations to travelling front solutions. Arch. Ration. Mech. Anal., 65:335-361, 1977.

M. Freidlin and J. Gartner. On the propagation of concentration waves in periodic and random media. Sov. Math. Dokl. 20:1282-1286, 1979.

K. P. Hadeler and F. Rothe. Travelling fronts in nonlinear diffusion equations. J. Math. Biol., 2:251-263, 1975.

S. Heinze. Wave Solutions to Reaction-Diffusion Systems in Perforated Domains. Z. Anal. Anwendungen, 20:661-676, 2001.

J. Keener. Homogenization and propagation in the bistable equation. Phys. D, $136: 1-17,2000$.

J. Keener and T. Lewis. Wave-block in excitable media due to regions of depressed excitability. SIAM J. Appl. Math., 61:293-316, 2000.

T. Keitt, M. A. Lewis, and R. Holt. Allee effects, invasion pinning, and species' borders. Amer. Natur., 157:203-216, 2001.

V. LeBlanc and C. Roy. Forced translational symmetry-breaking for abstract evolution equations. J. Abstr. Differ. Equ. Appl., 4:16-43, 2013.

G. Maciel and F. Lutscher. How individual movement response to habitat edges affects population persistence and spatial spread. Amer. Natur., 182:42-52, 2013. 
G. Maciel and F. Lutscher. Allee effects and population spread in patchy landscapes. J. Biol. Dyn., 9:109-123, 2015.

J. Musgrave and F. Lutscher. Integrodifference equations in patchy landscapes II: population level consequences. J. Math. Biol., 69:617-658, 2014.

J. Musgrave, F. Lutscher, and A. Girard. Population spread in patchy landscapes under a strong Allee effect. Theor. Ecol., 8:313-326, 2015.

G. Nadin. Critical travelling waves for general heterogeneous one-dimensional reaction-diffusion equations. Ann. Inst. H. Poincaré Anal. Non Linéaire, 32:841-873, 2015.

C. Roy. The origin of wave blocking for a bistable reaction-diffusion equation : A general approach. Master's thesis, University of Ottawa, 2012.

B. Sandstede, A. Scheel, and C. Wulff. Dynamics of spiral waves on unbounded domains using center-manifold reductions. J. Differential Equations, 141:122$149,1997$.

N. Shigesada and K. Kawasaki. Biological Invasions : Theory and Practice. Oxford University Press, New York, 1997.

N. Shigesada, K. Kawasaki, and E. Teramoto. Traveling periodic waves in heterogeneous environments. Theoret. Population Biol., 30:143-160, 1986.

P. Turchin. Quantitative Analysis of Movement: Measuring and Modeling Population Redistribution in Animals and Plants. Sinauer Associates, 1998.

A. I. Volpert, V.A. Volpert, and V.A. Volpert. Travelling Wave Solutions of Parabolic Systems, volume 140 of Translation of Mathematical Monographs. American Mathematical Society, Providence, R.I., 1994.

H. F. Weinberger. Long time behaviour of a class of biological models. SIAM J. Math. Anal., 13:353-396, 1982.

H. F. Weinberger. On spreading speeds and traveling waves for growth and migration models in a periodic habitat. J. Math. Biol., 45:511-548, 2002.

K. With. The landscape ecology of invasive spread. Conservation Biology, 16:1192-1203, 2000.

J.X. Xin. Existence and nonexistence of traveling waves and reaction-diffusion front propagation in periodic media. J. Statist. Phys., 73:893-926, 1993.

J. Xin. Front propagation in heterogeneous media. SIAM Rev., 42:161-230, 2002. 